\title{
Activity of Serotonergic Neurons in Behaving Animals
}

\author{
Barry L. Jacobs, Ph.D., and Casimir A. Fornal, Ph.D.
}

Brain serotonergic neurons display a distinctive slow and regular discharge pattern in behaving animals. This activity gradually declines across the arousal-waking sleep cycle, becoming virtually silent during rapid eye movement sleep. The activity of these neurons, in both the pontine and medullary groups, is generally unresponsive to a variety of physiological challenges or stressors. However, these neurons are activated in association with increased muscle tone/tonic motor activity, especially if the motor activity is in the repetitive or central pattern generator mode. We interpret these data within the following theoretical framework. The primary function of the brain serotonergic system is to facilitate motor output. Concurrently, the system coordinates autonomic and neuroendocrine function with the present motor demand, and inhibits information processing in various sensory pathways. Reciprocally, when the serotonin system is briefly inactivated (e.g., during orientation to salient stimuli), this disfacilitates motor function and disinhibits sensory information processing. It is within this context that serotonin exerts its well-known effects on pain, feeding, memory, mood, etc.

[Neuropsychopharmacology 21:9S-15S, 1999] (C) 1999 American College of Neuropsychopharmacology. Published by Elsevier Science Inc.
KEY WORDS: Serotonin; Neuronal activity; Cats; Raphe; Motor activity; Stress

Experience over the past 40 years in the field of neuroscience has taught us that analyzing neuronal activity (extracellularly recorded action potentials or single-unit activity) represents one of the most important methods for elucidating the functional or behavioral significance of brain systems in experimental animals. Research in this area was inspired by the pioneering work of two laboratories. In the late 1950s and 1960s, a series of elegant single-unit studies by Hubel and Wiesel delineated the operating characteristics and function of visual system neurons in cats and monkeys. Also in the 1960s, Evarts demonstrated the value of adapting this technique to behaving animals in order to study the

From the Program in Neuroscience, Department of Psychology, Princeton University, Princeton, New Jersey.

Address correspondence to: Dr. B.L. Jacobs, Program in Neuroscience, Department of Psychology, Green Hall, Princeton University, Princeton, NJ 08544.

Received November 5, 1998; revised January 20, 1999; accepted January 20, 1999. function of various structures within the motor system of monkeys. Our work in this area was made feasible by an extraordinary series of studies by Aghajanian in the late 1960s and early 1970s. He and his colleagues demonstrated that one could identify and reliably record the single-unit activity of brain serotonergic (5-HT), noradrenergic, and dopaminergic neurons in rats.

Single-unit studies of the brain 5-HT system in behaving animals, including our own, have been conducted almost exclusively in the domestic cat. In this article we review the past 20 years of our research in this area, including our most recent studies of the activity of medullary 5-HT neurons in relation to autonomic regulation. Finally, we propose a theoretical framework for interpreting these data, and indicate how our results are compatible with the rest of this field.

\section{BASIC ANATOMY}

The 5-HT system is primitive in a number of different ways (Jacobs and Azmitia 1992). First, the basic plan for 
the cell bodies of these neurons is conserved throughout the vertebrates. This implies that its function must, to some degree, be common to the physiology and behavior of fish, amphibians, and reptiles as well as mammals, including primates. Second, virtually all of the cell bodies of 5-HT neurons are found in the brain stem, on or near the midline. This implies a somewhat stronger involvement in basic processes, especially those associated with axial functions, such as controlling the trunk and proximal limb muscles. Finally, these neurons and their processes are among the first to develop with ontogeny. All of this suggests that the central 5-HT system subserves basic or fundamental functions within the CNS, but does not imply that it is limited to this (see below).

In vertebrates, the cell bodies of the majority of brain 5-HT neurons are localized in or near the brain stem raphe nuclei (Jacobs and Azmitia 1992). These midline clusters can be divided into two major groups (Figure 1). The rostral or superior group, localized in the pons/ mesencephalon, contains the two nuclei which supply most of the 5-HT to the forebrain: the nucleus centralis superior (NCS) and dorsal raphe nucleus (DRN). The caudal or inferior group, localized in the medulla, contains the three nuclei which supply most of the 5-HT to the spinal cord: the nucleus raphe magnus (NRM), nucleus raphe obscurus (NRO), and nucleus raphe pallidus (NRP).

We have examined the single-unit activity of neurons in both of these groups. In many ways their activity is quite similar, but they also differ in some interesting ways that reflects their different functional roles.

\section{NEURONAL ACTIVITY ACROSS THE SLEEP-WAKE-AROUSAL CYCLE}

During the quiet waking state in the cat, the activity of DRN 5-HT neurons is slow ( $\sim 3$ spikes/s) and regular, characteristic of the unmodulated operation of an endogenous pacemaker (Jacobs and Fornal 1991). During an aroused state or in response to sensory stimuli, neuronal activity can be increased $30 \%-50 \%$ above the quiet waking level. This is mediated at least in part by a glutamatergic input (Levine and Jacobs 1992). The most dramatic changes in the activity of 5-HT neurons occur during sleep. As animals become drowsy and enter slow-wave sleep, neuronal activity slows to approximately $50 \%$ of the quiet waking level and loses its regularity. This decline is mediated by activation of an inhibitory GABAergic input (Levine and Jacobs 1992; Nitz and Siegel 1997). Finally, during REM sleep, the activity of most brain 5-HT neurons falls to zero. This complete suppression of activity is mediated at least in part by GABA. This general pattern of activity across the sleep-wake-arousal cycle displayed by $5-\mathrm{HT}$ neurons in the DRN is also seen in the other major groups of brain stem 5-HT neurons: NCS, NRM, NRO, and NRP (Jacobs and Fornal 1991). There are, however, some interesting differences. For example, NRO/NRP serotonergic neurons tend to have a higher spontaneous discharge rate ( $\sim 5-6$ spikes/s during quiet waking) and their activity displays a shallow rate of decline in going from aroused waking through slow-wave sleep, but then also declines precipitously in REM sleep.

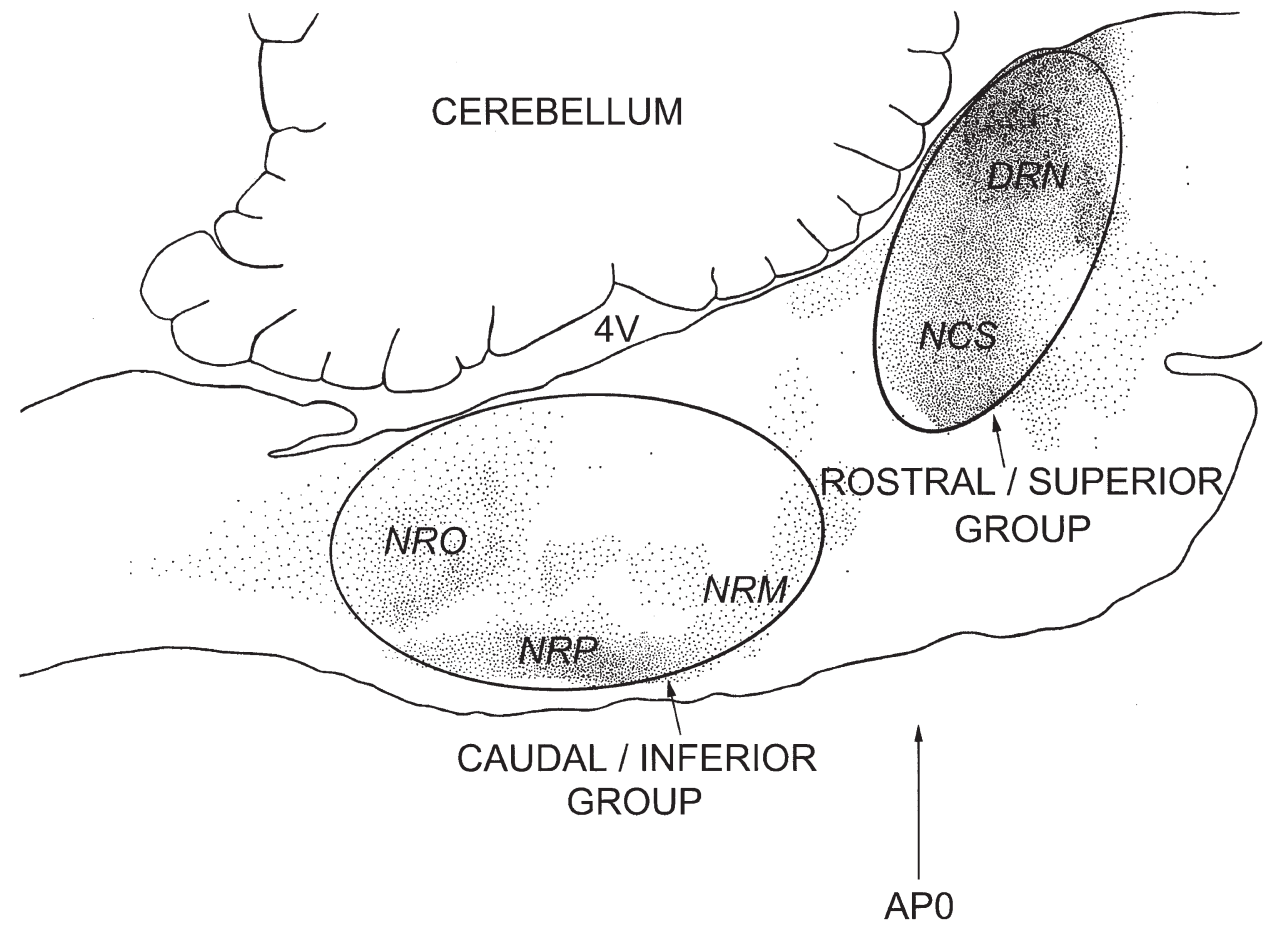

Figure 1. Midsaggital view of the cat brain stem with serotonin-immunoreactive cell bodies indicated by small dots. The ovals encompass the two major subdivisions of the brain serotonergic system. Abbreviations: $4 \mathrm{~V}$, fourth ventricle; $\mathrm{AP} 0$, stereotaxic zero in the anteriorposterior plane. 


\section{NEURONAL RESPONSE TO STRESSORS}

In attempting to understand the role of brain 5-HT neurons in physiology and behavior, we reasoned that it would be important to examine their activity under extreme conditions representing a variety of categories. Also, a number of studies have strongly implicated $5-\mathrm{HT}$ in the stress response. Thus, we examined neuronal responses in cats exposed to a variety of strong environmental and physiological stimuli (Auerbach et al. 1985; Fornal et al. 1987, 1989, 1990; Wilkinson and Jacobs 1988). Accordingly, while recording the activity of 5-HT neurons in the DRN, NCS, or NRM, cats were exposed to the following conditions: a heated environment or a pyrogen, drug-induced increases or decreases in blood pressure, insulin-induced hypoglycemia, phasic or tonic painful stimuli, systemic injections of morphine, loud noise, physical restraint, or a natural enemy (dog). Despite the fact that all of these conditions evoked strong behavioral responses and/or physiological changes indicative of sympathetic activation, none of them significantly activated 5-HT neuronal activity beyond the level typically seen during an undisturbed active waking state. A few examples from this series of studies illustrate this point.

The activity of DRN 5-HT neurons was examined in response to both increased ambient temperature and pyrogen-induced fever, stimuli eliciting opposite thermoregulatory responses (Fornal et al. 1987). Neuronal activity remained unaffected as ambient temperature was increased from $25^{\circ} \mathrm{C}$ to $43^{\circ} \mathrm{C}$. Following prolonged heat exposure, cats displayed intense continuous panting, relaxation of posture, and a progressive rise in body/brain temperature (range $0.5^{\circ} \mathrm{C}-2.0^{\circ} \mathrm{C}$ ), yet no change in 5-HT neuronal activity occurred. In a parallel study, a synthetic pyrogen (muramyl dipeptide) was administered systemically resulting in increased body/ brain temperature within 30 minutes and lasting for approximately 6 hours. The peak elevation of body temperature was typically $1.5^{\circ} \mathrm{C}-2.5^{\circ} \mathrm{C}$, yet, once again, no change in neuronal activity was observed. Consistent with these electrophysiological results, we have also found no change in extracellular levels of 5-HT in the anterior hypothalamus, a primary thermoregulatory center of the cat, following pyrogen administration (Wilkinson et al. 1991).

A large and relatively consistent body of evidence implicates central 5-HT in analgesia, especially those 5-HT neurons localized in the NRM and projecting to the dorsal horn. Accordingly, we examined the activity of NRM 5-HT neurons in behaving cats exposed to a variety of phasic or tonic painful stimuli. No change in neuronal activity was produced by these stimuli relative to the discharge rate during an undisturbed active waking baseline (Auerbach et al. 1985). There was also no change in 5-HT neuronal activity in response to the systemic administration of morphine, in a dose that produced analgesia. These results have recently been confirmed in studies reporting that identified NRM 5-HT neurons in the rat were not activated by painful stimuli eliciting the withdrawal reflex or by analgesic doses of morphine (Potrebic et al. 1994; Gao et al. 1998).

Our studies employing in vivo brain microdialysis measures of extracellular 5-HT release in rats also support this general conclusion. We found no increase, relative to an active waking baseline, in forebrain 5-HT levels in rats exposed to: painful stimuli; forced swimming; or a natural enemy (cat) (Rueter and Jacobs 1996).

In summary, these data indicate that mild to relatively strong stressors, drawn from a number of different environmental and physiological categories, do not significantly perturb the rostral group of brain stem serotonergic neurons when compared to a non-stressful, but attentive and active waking state. In the Discussion section we speculate on how these results can be reconciled with the large literature indicating that 5-HT is importantly involved in the organisms' responses to pain and stress.

\section{MUSCLE TONE/TONIC MOTOR ACTIVITY}

Paralysis, produced by inhibition of motoneurons controlling antigravity muscle tone, is a fundamental feature of REM sleep. Because the activity of most 5-HT neurons is almost totally suppressed during REM sleep, we examined the possibility that there might be a relationship between these two phenomena. Lesions of the dorsomedial pons produce a condition which permits investigation of this issue. Cats with a bilateral lesion here enter a stage of sleep which by all criteria appears to be REM sleep except that antigravity muscle tone is present and the animals are thus capable of movement and even coordinated locomotion (Jouvet and Delorme 1965).

During both waking and slow-wave sleep, the activity of DRN 5-HT neurons in these pontine-lesioned cats was similar to that in normal animals (Trulson et al. 1981). However, when these animals entered REM sleep, neuronal activity increased instead of displaying the decrease typical of this state. Those animals displaying the greatest amount of muscle tone and overt behavior during REM sleep showed the highest levels of neuronal activity, with some of their 5-HT neurons discharging at a level approximating that of the waking state.

This experiment led us to explore this issue from a conceptually related, but methodologically different, perspective. If carbachol, a cholinomimetic agent, is micro-injected into this same dorsolateral pontine area, a condition somewhat reciprocal to non-atonia REM sleep can be produced. These animals were awake, as 
demonstrated by their ability to track visual stimuli, but were otherwise paralyzed (Steinfels et al. 1983). However, their DRN 5-HT neurons were inactive. In the same study, we also found that a centrally acting muscle relaxant completely suppressed 5-HT neuronal activity, but peripheral neuromuscular block had no effect on 5-HT neuronal activity. This latter finding suggests that although 5-HT neurons influence motor activity they are essentially unresponsive to afferent feedback deriving from these same muscles. Recently, we have extended these carbachol experiments to NRO/NRP 5-HT neurons and have obtained similar results to those just described for DRN neurons (unpublished observations).

\section{PHASIC MOTOR ACTIVITY}

More recently we have observed additional, and much more specific, relationships between 5-HT neuronal activity and motor function. When cats engage in a variety of types of central pattern generator mediated oralbuccal activities, such as chewing/biting, licking, or grooming, approximately one-fourth of DRN and NCS 5 -HT neurons increase their activity by as much as $2-$ 5 -fold (Fornal et al. 1996). In contrast, the rest of the 5-HT neurons in these nuclei maintain their slow and rhythmic activity. These increases in neuronal activity often precede the initiation of movement by several seconds, but they invariably terminate coincident with the end of the behavioral sequence. Equally impressive is the fact that even brief (1-5 seconds) spontaneous pauses in these ongoing patterned behaviors are accompanied by an immediate decrease in 5-HT neuronal activity to, or below, baseline levels. The increased neuronal activity during these central pattern generatormediated behaviors is typically tonic, and regular, lasting for the duration of the expressed behavior. During a variety of other episodic or purposive movements, even those involving oral-buccal responses, such as yawning, no increase, or even a decrease, in 5-HT neuronal activity is seen.

Most of these DRN neurons can also be activated by somatosensory stimuli applied to the head and neck region, while the same stimuli applied to the rest of the body surface are typically ineffective. Somewhat surprisingly, the level of increased activity produced by somatosensory stimulation often approximates that seen spontaneously during oral-buccal movements (i.e., 25-fold).

There is often an inverse relationship between the activity of 5-HT neurons and the occurrence of strong attentional shifts. In fact, during orienting responses to novel or imperative stimuli, the activity of DRN and NCS 5-HT neurons may fall silent for several seconds.
As described above, 5-HT neurons in the rostral pons (DRN and NCS) provide almost the entire 5-HT innervation of the forebrain, whereas those in the caudal medulla (NRP and NRO) are the source of much of the 5-HT innervation of the spinal cord, especially the ventral horn. Therefore, it is of some interest to compare the response properties of neurons in these two disparate groups.

Contrary to pontine 5-HT neurons, where only a subgroup of neurons are activated during central pattern generator-mediated behaviors, virtually all of the medullary 5-HT neurons that we examined were activated under at least some of these conditions (Veasey et al. 1995). The degree of activation, however, is much less impressive (i.e., $50 \%-100 \%$ versus $100 \%-400 \%$ for the DRN and NCS). In this context, it may be important to recall that the basal quiet waking discharge rate of the medullary 5-HT neurons is approximately twice that of the pontine 5-HT neurons, 5-6 spikes/s versus $2-3$ spikes/s. There appears to be at least some degree of response specificity in these neurons. Thus, virtually all medullary 5-HT neurons are activated during treadmill-induced locomotion, but only subgroups are activated during hyperpnea (induced by exposure to $\mathrm{CO}_{2}$ ), or during feeding. Many of the 5-HT neurons are activated in association with more than one of these motor activities. In most cases there is a strong positive correlation between magnitude of neuronal activation and speed of locomotion and/or depth of respiration. As with pontine 5-HT neurons the increased activity of medullary 5-HT neurons is tightly coupled to the onset and offset of the behavior. Unlike pontine 5-HT neurons, medullary 5-HT neurons do not appear to be activated by somatosensory stimuli applied to any region of the body surface. Nor is their activity significantly changed during orientation to strong or novel stimuli. Finally, when DRN and NCS neurons were examined under the same conditions, none of them were activated during treadmill-induced locomotion, and only a small number were weakly activated during $\mathrm{CO}_{2}-$ induced hyperpnea (Veasey et al. 1997). As described above, a subgroup of pontine 5-HT neurons were strongly activated during feeding.

\section{RELATION TO SYMPATHETIC ACTIVITY}

More recently, we have turned our attention to examining the relationship between sympatho-adrenal activation and the activity of caudal raphe (NRO/NRP) 5-HT neurons. These neurons are of especial interest in this context because their axons descend into the spinal cord and often collateralize to the intermediolateral column.

In one series of experiments (Martín et al. 1998), we examined the activity of 5-HT NRO/NRP neurons during 
manipulations of the cardiovascular system. Transient alterations in arterial blood pressure (and sympathetic activity) were produced by intravenous administration of vasoactive drugs. The activity of 5-HT NRO/NRP neurons was not significantly altered in response to phenylephrine (a vasoconstrictor) or sodium nitroprusside (a vasodilator), at doses which elicited marked reflex bradycardia and tachycardia, respectively. Furthermore, no significant changes in neuronal activity were observed after systemic administration of hydralazine, a direct long-acting vasodilator, despite prolonged reflex activation of sympathetic outflow, as indicated by increases in heart rate and plasma norepinephrine levels. Acute venous hemorrhage, up to $22.5 \%$ of estimated total blood volume, also had no effect on the activity of $\mathrm{NRO/NRP} \mathrm{neurons,} \mathrm{although} \mathrm{plasma} \mathrm{catecholamine}$ levels were significantly elevated by this manipulation. Thus, these results do not support a direct role of 5-HT $\mathrm{NRO} / \mathrm{NRP}$ neurons in the reflex alterations in sympathetic (and parasympathetic) outflow invoked by these cardiovascular manipulations.

In a subsequent experiment (Jacobs et al. 1997), insulin-induced hypoglycemia was used to preferentially activate the sympatho-adrenal system. Following insulin administration, plasma epinephrine levels increased by as much as 8-fold. Surprisingly, the discharge rate of 5-HT NRO/NRP neurons was reduced by approximately $40 \%$ after insulin administration. The subsequent administration of glucose, at doses sufficient to reverse hypoglycemia, promptly restored neuronal activity and plasma catecholamines to baseline levels. Because NRO/NRP 5-HT neurons are thought to subserve a sympathoexcitatory role, these results are the opposite of what would be predicted for these cells. However, animals treated with insulin also displayed diminished skeletal muscle tone and signs of muscle weakness, which closely paralleled the decreases in neuronal activity observed following insulin administration. Thus, the effect of systemic insulin on 5-HT $\mathrm{NRO} / \mathrm{NRP}$ neuronal activity may be related more closely to changes in motor output than to changes in sympathetic outflow. Interestingly, 5-HT neurons in the rostral DRN were previously found to be unaffected by this manipulation (Fornal et al. 1989).

In another study (Martín et al. 1997), we examined the effect of cold stress, a potent activator of the sympathetic nervous system. The discharge rate of approximately half of all NRO/NRP cells studied was significantly increased during exposure to a low ambient temperature $\left(5^{\circ} \mathrm{C}\right)$, which induced shivering and piloerection in animals, but no appreciable changes in core temperature. The elevation in neuronal activity was maintained throughout the entire cooling period (4 hours) and was unrelated to the occurrence of shivering bursts per se. Although these results suggest that some NRO/NRP 5-HT neurons may play a role in the physio- logical responses underlying cold defense (e.g., increased sympathetic outflow), it is unclear whether the primary stimulus to these neurons was sympathetic or motor in nature. In addition, there appears to be some specificity of the neuronal response to cold exposure, since the activity of DRN 5-HT neurons was unaffected by this manipulation.

Overall, these results suggest that the discharge of 5-HT NRO/NRP neurons is not strongly related to sympathetic activity in the absence of concomitant changes in motor output. These neurons may indeed influence sympathetic activity, but do so only in relation to their primary role in motor control. Thus, the function of these neurons may be to modulate the activity of both sympathetic and somatic motor systems in accordance with the level of motor output.

\section{DISCUSSION}

These data indicate that, with the exception of motor activity, the discharge rate of brain 5-HT neurons during the waking state is regulated within fairly tight limits. This is supported by our rat brain microdialysis studies showing that a variety of conditions (both stressful and non-stressful) produce approximately the same magnitude of change in brain 5-HT and, furthermore, do so in a number of different forebrain sites (Rueter and Jacobs 1996). It is important to emphasize that stressors do activate the serotonergic system, but that they do so no more than other non-stressful, activating conditions. One way of reconciling this relative invariance with the vast array of behavioral and physiological processes with which 5-HT has been implicated is to invoke a concept that might be termed conjunctive activation. Thus, 5-HT released during feeding may exert a role in satiety only in the presence of increased release of cholecystokinin (CCK). Using this argument, 5-HT released in reaction to exposure to stressors would exert a different effect than it does during feeding because it now interacts with corticosterone rather than CCK. In addition, this acknowledges that such interactions may occur only in specific brain areas (e.g., hypothalamus versus hippocampus). Posting that serotonin is mostly closely linked to motor activity and autonomic regulation is not incompatible with its exerting important roles in feeding, analgesia, or memory. It simply directs us to envision how such processes are interrelated, for example, analgesia and locomotor activity or memory and autonomic function.

There is a general relationship between level of tonic motor activity and 5-HT neuronal activity across all groups of 5-HT neurons. Superimposed upon this in some neurons is an additional relationship in which a further, often dramatic, activation is seen in association 
with repetitive, central pattern generator-mediated behaviors. We interpret these data within the following framework. The primary function of this increased 5-HT neuronal activity in association with tonic and repetitive motor output is to facilitate motor function, and secondarily to coordinate autonomic and neuroendocrine function in association with the existing motor demand, and to suppress activity in most sensory information-processing channels (Figure 2).

Reciprocally, during the active inhibition of gross behavior (e.g., during orientation), the activity of a subgroup of pontine 5-HT neurons is suppressed, thus disfacilitating motor output and disinhibiting sensory information processing.

There are several reasons for believing that the facilitation of motor output by $5-\mathrm{HT}$ is general in nature. First, 5-HT neuronal activity is generally tonically elevated during the execution of a particular behavior, suggesting that 5 -HT is facilitating the "behavior" rather than a particular motor act or muscle group that is a component of the behavior. Second, the distribution of 5-HT axon terminal innervation of the brain stem and spinal cord is consistent with the involvement of 5-HT in patterned movement employing gross skeletal muscles rather than movements using finer or more discrete muscles, such as those controlling the eyes or the digits. Thus, in the spinal cord there is a denser input to the medial portion of the ventral horn, where axial motoneurons serving the trunk and limbs are found, compared to the lateral portions, where distal motoneurons serving paws and digits are found (Steinbusch 1981). Third, there is evidence that spinally projecting medullary 5-HT neurons collateralize at various levels of the cord, suggesting a broad relationship to motor output (Skagerberg and Bjorklund 1985). Fourth, the fact that single medullary 5-HT neurons may project to both the intermediolateral column of the spinal cord as well as to the ventral horn suggests an integrative function involving motor and autonomic control (Allen and Cechetto 1994).

Finally, we speculate on how these data may bear upon issues regarding human psychopathology. It is well known that a variety of drugs that elevate synaptic levels of 5-HT are useful in the treatment of a number of pathologies, including depression and obsessive-compulsive disorders. Therefore, it might be beneficial to train depressed patients to jog or ride a stationary bicycle. In doing so, our data indicate that they could directly increase their synaptic level of 5-HT in a biological, rather than a pharmacological, manner. Such a regimen might serve as a useful adjunct to pharmacotherapy. Exercise may lack the effectiveness of primary antidepressant treatments, such as SSRIs and MAOIs, because it does not block the inactivation of serotonin. We also believe that patients with obsessive-compulsive disorder may be engaging in repetitive or compulsive motor acts (or thought patterns) as a means of activating their brain 5-HT system in a nonpharmacological manner for some beneficial or rewarding effect. Treating them with drugs may accomplish the same brain neurochemical endpoint and thereby allow them to stop engaging in time consuming, socially unacceptable, and often harmful behaviors.

Our hypothesis suggests that the behavior we engage in can change brain function. This is, of course, the reverse of the way we traditionally think of this relationship (i.e., that changes in the brain lead to changes in behavior). This also raises the important issue of why the manipulation of a system that is primarily associated with motor activity has profound mood altering effects. It suggests that an as yet unexplored general link may exist between mood and motor activity.

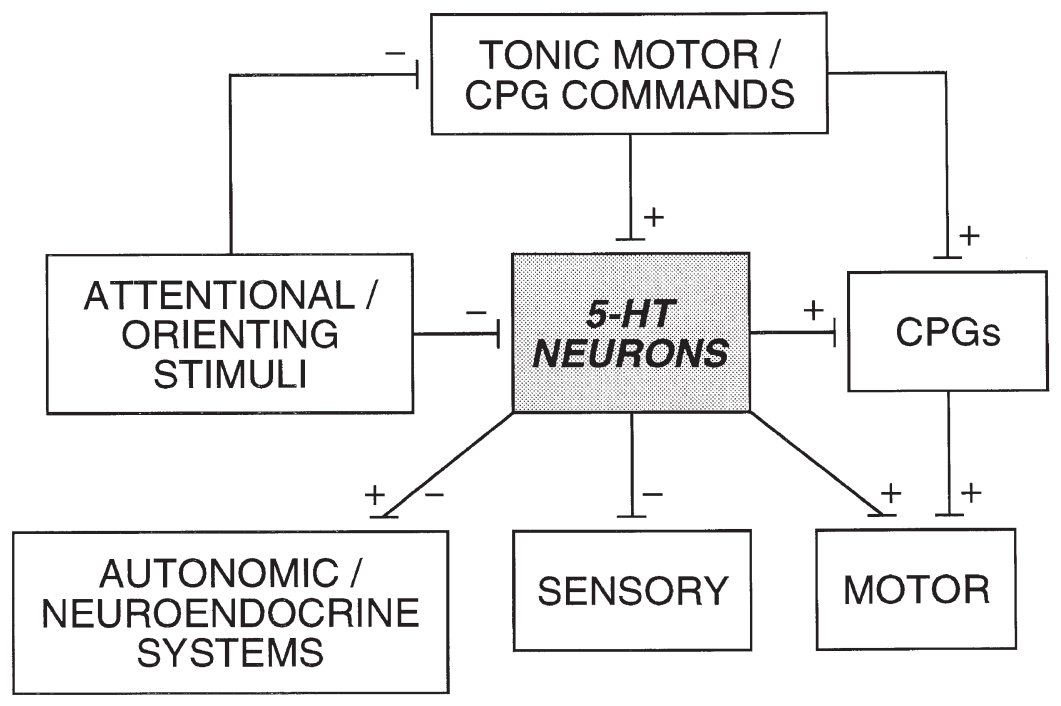

Figure 2. Schematic representation of the major components of the motor hypothesis of serotonergic function. Serotonergic neurons (5-HT neurons) are activated in association with motor comands which also activate central pattern generators (CPGs), which in turn activate motoneurons. Once activated, serotonergic neurons exert influences on neuroendocrine and autonomic output, sensory information processing, and upon CPGs and motoneurons. In addition, the activity of serotonergic neurons can be phasically suppressed in association with stimuli eliciting attentional or orienting responses, which also suppress tonic motor commands. (Jacobs and Fornal 1995) (Adapted from Semin Neurosci (1995) 7:401-408.) 


\section{REFERENCES}

Allen GV, Cechetto DF (1994): Serotoninergic and nonserotoninergic neurons in the medullary raphe system have axon collateral projections to autonomic and somatic cell groups in the medulla and spinal cord. J Comp Neurol 350:357-366

Auerbach S, Fornal C, Jacobs BL (1985): Response of serotonin-containing neurons in nucleus raphe magnus to morphine, noxious stimuli, and periaqueductal gray stimulation in freely moving cats. Exp Neurol 88:609-628

Fornal CA, Litto WJ, Morilak DA, Jacobs BL (1987): Singleunit responses of serotonergic dorsal raphe nucleus neurons to environmental heating and pyrogen administration in freely moving cats. Exp Neurol 98:388-403

Fornal CA, Litto WJ, Morilak DA, Jacobs BL (1989): Singleunit responses of serotonergic neurons to glucose and insulin administration in behaving cats. Am J Physiol 257:R1345-R1353

Fornal CA, Litto WJ, Morilak DA, Jacobs BL (1990): Singleunit responses of serotonergic dorsal raphe neurons to vasoactive drug administration in freely moving cats. Am J Physiol 259:R963-R972

Fornal CA, Metzler CW, Marrosu F, Ribiero-do-Valle LE, Jacobs BL (1996): A subgroup of dorsal raphe serotonergic neurons in the cat is strongly activated during oralbuccal movements. Brain Res 716:123-133

Gao K, Chen DO, Genzen JR, Mason P (1998): Activation of serotonergic neurons in the raphe magnus is not necessary for morphine analgesia. J Neurosci 18:1860-1868

Jacobs BL, Fornal CA (1991): Activity of brain serotonergic neurons in the behaving animal. Pharmacol Rev 43:563578

Jacobs BL, Azmitia EC (1992): Structure and function of the brain serotonin system. Physiol Rev 72:165-229

Jacobs BL, Fornal CA (1995): Activation of 5-HT neuronal activity during motor behavior. Semin Neurosci 7:401-408

Jacobs BL, Martín FJ, Fornal CA, Metzler CW (1997): Systemic administration of insulin decreases the activity of medullary serotonergic neurons in awake cats. Soc Neurosci Abstr 23:1227

Jouvet M, Delorme F (1965): Locus coeruleus et sommeil paradoxal. C R Soc Biol 159:895-899

Levine ES, Jacobs BL (1992): Neurochemical afferents controlling the activity of serotonergic neurons in the dorsal raphe nucleus: Microiontophoretic studies in the awake cat. J Neurosci 12:4037-4044
Martín FJ, Gallegos RA, Fornal CA, Metzler CW, Jacobs BL (1997): Effect of environmental cooling on the activity of pontine and medullary serotonergic neurons in awake cats. Soc Neurosci Abstr 23:1226

Martín FJ, Fornal CA, Metzler CW, Jacobs BL (1998): The activity of medullary serotonergic neurons in freely moving cats is unaffected by cardiovascular manipulations that evoke reflex changes in autonomic outflow. Soc Neurosci Abstr 24:1106

Nitz D, Siegel J (1997): GABA release in the dorsal raphe nucleus: Role in the control of REM sleep. Am J Physiol 273:R451-R455

Potrebic SB, Field HL, Mason P (1994): Serotonin immunoreactivity is contained in one physiological cell class in the rat rostral ventromedial medulla. J Neurosci 14:1655-1665

Rueter LE, Jacobs BL (1996): A microdialysis examination of serotonin release in the rat forebrain induced by behavioral/environmental manipulations. Brain Res 739:57-69

Skagerberg G, Bjorklund A (1985): Topographic principles in the spinal projections of serotonergic and non-serotonergic brainstem neurons in the rat. Neurosci 15:445-480

Steinbusch HWM (1981): Distribution of serotonin-immunoreactivity in the central nervous system of the ratCell bodies and terminals. Neuroscience 4:557-618

Steinfels GF, Heym J, Strecker RE, Jacobs BL (1983): Raphe unit activity in freely moving cats is altered by manipulations of central but not peripheral motor systems. Brain Res 279:77-84

Trulson ME, Jacobs BL, Morrison AR (1981): Raphe unit activity during REM sleep in normal cats and in pontine lesioned cats displaying REM sleep without atonia. Brain Res 226:75-91

Veasey SC, Fornal CA, Metzler CW, Jacobs BL (1995): Response of serotonergic caudal raphe neurons in relation to specific motor activities in freely moving cats. J Neurosci 15:5346-5359

Veasey SC, Fornal CA, Metzler CW, Jacobs BL (1997): Singleunit responses of serotonergic dorsal raphe neurons to specific motor challenges in freely moving cats. Neuroscience 79:161-169

Wilkinson LO, Auerbach SB, Jacobs BL (1991): Extracellular serotonin levels change with behavioral state but not pyrogen-induced hyperthermia. J Neurosci 11:27322741

Wilkinson LO, Jacobs BL (1988): Lack of response of serotonergic neurons in the dorsal raphe nucleus of freely moving cats to stressful stimuli. Exp Neurol 101:445-457 